Yang Liguo*, Wang Xin, Niu Yongsheng, Song Haixiang, Tian Dayong and Gao Linna

\title{
Crystal structure of catena-poly[aqua-( $\mu_{2}-1,7$-dicarba-closo-
} dodecaborane-1,7-dicarboxylato- $\left.\mathrm{K}^{2} O: O^{\prime}\right)$-(1,10-phenanthrolin- $\left.\mathrm{K}^{2} N, N^{\prime}\right)$
copper(II)], $\mathrm{C}_{16} \mathrm{H}_{20} \mathrm{~B}_{10} \mathrm{CuN}_{2} \mathrm{O}_{5}$

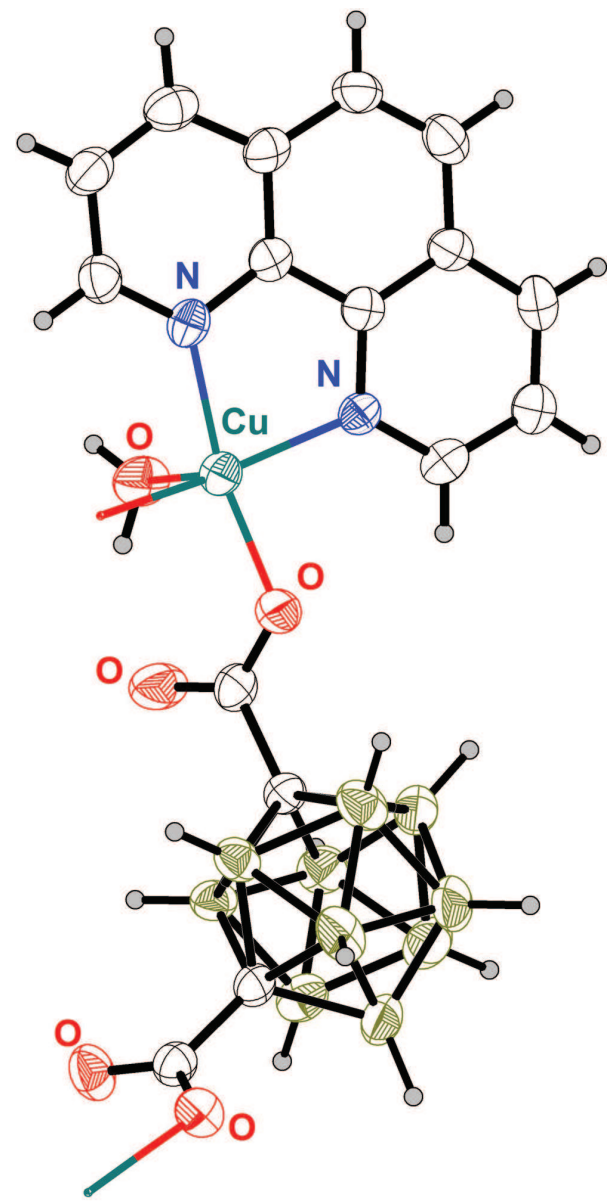

https://doi.org/10.1515/ncrs-2018-0351

Received September 4, 2018; accepted November 30, 2018; available online December 19, 2018

Abstract
$\mathrm{C}_{16} \mathrm{H}_{20} \mathrm{~B}_{10} \mathrm{CuN}_{2} \mathrm{O}_{5}$, monoclinic, $P 2_{1} / n($ no. 14$), a=7.4252(8) \AA$,
$b=13.5409(14) \AA, \quad c=23.020(3) \AA, \quad \beta=94.4880(10)^{\circ}$,

*Corresponding author: Yang Liguo, College of Chemistry and Environmental Engineering, Anyang Institute of Technology, Anyang 455000, Henan, P.R. China, e-mail: lgyang@ayit.edu.cn

Wang Xin, Niu Yongsheng, Song Haixiang and Tian Dayong: College of Chemistry and Environmental Engineering, Anyang Institute of Technology, Anyang 455000, Henan, P.R. China

Gao Linna: College of Chemical and Environmental Engineering, Shandong University of Science and Technology, Qingdao 266590, P.R. China

๖ Open Access. ๑ 2019 Yang Liguo et al., published by De Gruyter. (c) BY License.
$V=2307.4(4) \AA^{3}, Z=4, R_{\text {gt }}(F)=0.0583, w R_{\text {ref }}\left(F^{2}\right)=0.1320$, $\mathrm{T}=298(2) \mathrm{K}[1-3]$.

CCDC no.: 1881270

Table 1: Data collection and handling.

\begin{tabular}{ll}
\hline Crystal: & Blue block \\
Size: & $0.12 \times 0.10 \times 0.08 \mathrm{~mm}$ \\
Wavelength: & Mo $K \alpha$ radiation $(0.71073 \AA)$ \\
$\mu:$ & $0.98 \mathrm{~mm}^{-1}$ \\
Diffractometer, scan mode: & $\mathrm{CCD}, \varphi$ and $\omega$ \\
$\theta_{\max }$, completeness: & $25.0^{\circ},>99 \%$ \\
$N(h k l)_{\text {measured }}, N(h k l)_{\text {unique }}, R_{\text {int }}:$ & $13596,4058,0.108$ \\
Criterion for $I_{\text {obs }}, N\left(h k l_{\text {gt }}:\right.$ & $I_{\text {obs }}>2 \sigma\left(l_{\text {obs }}\right), 1934$ \\
$N(\text { param })_{\text {refined }}:$ & 307 \\
Programs: & Bruker $[1]$, SHELX $[2,3]$ \\
\hline
\end{tabular}

The crystal structure is shown in the figure. Tables 1 and 2 contain details on crystal structure and measurement conditions and a list of the atoms including atomic coordinates and displacement parameters.

\section{Source of material}

Solvents, which were employed to prepare the title complexes, were dried with appropriate drying agents and distilled under nitrogen prior to use. Dichloromethane was directly used without further purification. The 1,7dihydroxycarbonyl-1,7-dicarba-closo-dodecaborane was prepared according to the literature [4].

$\mathrm{Cu}(\mathrm{OAc})_{2} \cdot \mathrm{H}_{2} \mathrm{O}(34.1 \mathrm{mg}, 0.2 \mathrm{mmol})$ was added to a suspension of 1,7-(COOH $)_{2}-1,7-\mathrm{C}_{2} \mathrm{~B}_{10} \mathrm{H}_{10} \quad(0.10 \mathrm{mmol})$ and 1,10-phenanthroline $(37.0 \mathrm{mg}, 0.2 \mathrm{mmol})$ in chloromethane $(10 \mathrm{~mL})$. The mixture was stirred for $6 \mathrm{~h}$ at room temperature under the protection of dry nitrogen. Then a colourless solution formed. Crystals were obtained by slow evaporation of dichloromethane-n-hexane (34.4 mg, 20.9\%). m.p. 188-189 K. FTIR (KBr) $v\left(\mathrm{~cm}^{-1}\right)$ : 2980, 2960, 2920, $2865(\mathrm{C}-\mathrm{H})$; 2610, 2600, 2582, 2552 (B-H).

\section{Experimental details}

All $\mathrm{H}$ atoms were placed geometrically and treated as riding on their parent atoms, with $\mathrm{B}-\mathrm{H} 1.10, \mathrm{C}-\mathrm{H} 0.96$, with $U_{\text {iso }}(\mathrm{H})=1.2 U_{\text {eq }}(\mathrm{B}), U_{\text {iso }}(\mathrm{H})=1.5 U_{\text {eq }}(\mathrm{C})$. 
Table 2: Fractional atomic coordinates and isotropic or equivalent isotropic displacement parameters $\left(\AA^{2}\right)$.

\begin{tabular}{|c|c|c|c|c|}
\hline Atom & $x$ & $y$ & $z$ & $U_{\text {iso }} * / U_{\text {eq }}$ \\
\hline Cu1 & $0.62348(9)$ & $0.89743(4)$ & $0.85985(3)$ & $0.0483(2)$ \\
\hline N1 & $0.6945(6)$ & $1.0407(3)$ & $0.8666(2)$ & $0.0507(12)$ \\
\hline $\mathrm{N} 2$ & $0.6744(5)$ & $0.9041(3)$ & $0.94820(17)$ & $0.0435(11)$ \\
\hline 01 & $0.5328(5)$ & $0.7641(3)$ & $0.86750(15)$ & $0.0582(11)$ \\
\hline 02 & $0.6874(6)$ & $0.6861(3)$ & $0.8024(2)$ & $0.0846(14)$ \\
\hline 03 & $0.5140(5)$ & $0.9178(2)$ & $0.78020(15)$ & $0.0541(10)$ \\
\hline 04 & $0.2428(6)$ & $0.9233(3)$ & $0.81478(17)$ & $0.0697(12)$ \\
\hline 05 & $0.8944(5)$ & $0.8470(3)$ & $0.83441(16)$ & $0.0665(11)$ \\
\hline $\mathrm{H} 5 \mathrm{C}$ & 0.8643 & 0.7960 & 0.8140 & 0.080 * \\
\hline H5D & 0.9777 & 0.8777 & 0.8188 & 0.080 * \\
\hline B1 & $0.2359(10)$ & $0.6169(5)$ & $0.8675(3)$ & $0.066(2)$ \\
\hline $\mathrm{H} 1$ & 0.1835 & 0.6877 & 0.8825 & 0.079 * \\
\hline B2 & $0.4098(11)$ & $0.5534(5)$ & $0.9091(3)$ & $0.070(2)$ \\
\hline $\mathrm{H} 2$ & 0.4693 & 0.5833 & 0.9507 & $0.084^{\star}$ \\
\hline B3 & $0.5420(10)$ & $0.4888(5)$ & $0.8592(3)$ & $0.061(2)$ \\
\hline H3 & 0.6879 & 0.4772 & 0.8690 & $0.073^{*}$ \\
\hline B4 & $0.4492(9)$ & $0.5130(4)$ & $0.7883(3)$ & $0.0509(17)$ \\
\hline $\mathrm{H} 4$ & 0.5342 & 0.5162 & 0.7513 & $0.061^{*}$ \\
\hline B5 & $0.2604(9)$ & $0.5933(5)$ & $0.7931(3)$ & $0.0521(17)$ \\
\hline H5 & 0.2231 & 0.6489 & 0.7594 & 0.062 * \\
\hline B6 & $0.0943(10)$ & $0.5282(6)$ & $0.8287(3)$ & $0.065(2)$ \\
\hline $\mathrm{H} 6$ & -0.0510 & 0.5401 & 0.8180 & 0.078 * \\
\hline B7 & $0.1891(12)$ & $0.5027(6)$ & $0.9012(3)$ & $0.087(3)$ \\
\hline $\mathrm{H} 7$ & 0.1043 & 0.4986 & 0.9382 & $0.105^{*}$ \\
\hline B8 & $0.3792(12)$ & $0.4236(6)$ & $0.8961(3)$ & $0.078(3)$ \\
\hline $\mathrm{H} 8$ & 0.4187 & 0.3685 & 0.9299 & 0.094 * \\
\hline B9 & $0.4017(9)$ & $0.4005(5)$ & $0.8214(3)$ & $0.0591(19)$ \\
\hline H9 & 0.4539 & 0.3300 & 0.8059 & $0.071^{*}$ \\
\hline B10 & $0.1837(10)$ & $0.4083(5)$ & $0.8470(3)$ & $0.069(2)$ \\
\hline $\mathrm{H} 10$ & 0.0954 & 0.3433 & 0.8482 & $0.083^{*}$ \\
\hline $\mathrm{C} 1$ & $0.4442(7)$ & $0.6005(4)$ & $0.8412(2)$ & $0.0467(13)$ \\
\hline $\mathrm{C} 2$ & $0.2351(7)$ & $0.4685(4)$ & $0.7846(2)$ & $0.0461(14)$ \\
\hline C3 & $0.5689(8)$ & $0.6912(4)$ & $0.8365(3)$ & $0.0536(15)$ \\
\hline $\mathrm{C} 4$ & $0.3452(9)$ & $0.9326(4)$ & $0.7753(2)$ & $0.0501(15)$ \\
\hline C5 & $0.7106(8)$ & $1.1078(4)$ & $0.8245(2)$ & $0.0565(15)$ \\
\hline $\mathrm{H} 5 \mathrm{~A}$ & 0.6839 & 1.0891 & 0.7860 & 0.068 * \\
\hline C6 & $0.7662(8)$ & $1.2053(4)$ & $0.8366(3)$ & $0.0631(17)$ \\
\hline $\mathrm{H} 17$ & 0.7779 & 1.2494 & 0.8062 & $0.076^{*}$ \\
\hline $\mathrm{C} 7$ & $0.8031(8)$ & $1.2355(4)$ & $0.8924(3)$ & $0.0636(17)$ \\
\hline H11 & 0.8364 & 1.3007 & 0.9002 & $0.076^{*}$ \\
\hline $\mathrm{C} 8$ & $0.7908(7)$ & $1.1677(4)$ & $0.9389(3)$ & $0.0518(15)$ \\
\hline C9 & $0.7375(7)$ & $1.0702(4)$ & $0.9232(2)$ & $0.0432(13)$ \\
\hline C10 & $0.7264(7)$ & $0.9956(4)$ & $0.9673(2)$ & $0.0433(13)$ \\
\hline C11 & $0.7683(7)$ & $1.0192(4)$ & $1.0262(2)$ & $0.0479(14)$ \\
\hline C12 & $0.8195(8)$ & $1.1186(5)$ & $1.0415(3)$ & $0.0623(17)$ \\
\hline $\mathrm{H} 12$ & 0.8451 & 1.1351 & 1.0805 & $0.075^{*}$ \\
\hline C13 & $0.8312(8)$ & $1.1893(4)$ & $0.9997(3)$ & $0.0597(16)$ \\
\hline $\mathrm{H} 13$ & 0.8662 & 1.2529 & 1.0108 & $0.072^{*}$ \\
\hline C14 & $0.7563(8)$ & $0.9423(5)$ & $1.0667(2)$ & $0.0601(17)$ \\
\hline H14 & 0.7832 & 0.9538 & 1.1063 & $0.072^{*}$ \\
\hline C15 & $0.7042(8)$ & $0.8499(4)$ & $1.0473(3)$ & $0.0613(17)$ \\
\hline $\mathrm{H} 15$ & 0.6971 & 0.7984 & 1.0737 & $0.074^{*}$ \\
\hline C16 & $0.6621(8)$ & $0.8334(4)$ & $0.9881(3)$ & $0.0546(15)$ \\
\hline $\mathrm{H} 16$ & 0.6240 & 0.7708 & 0.9759 & $0.066^{*}$ \\
\hline
\end{tabular}

\section{Comment}

The polyhedral boron clusters "carboranes" have extensive applications in fundamental chemistry and applications as pharmaceuticals [5-8]. A series of amine-carboxyboranes and polyhedral hydroborate salts were tested for their antineoplastic-cytotoxic activity. Several carboxylate derivatives of $\mathrm{Cu}$ based on 1,2- or 1,7-dicarba-closo-dodecaborane have also been reported, in which the carborane cage is linked to the carboxylic moiety via a boron or carbon atom. Currently, the best known and most investigated carboranes are the three 12- vertex ortho, meta, and para isomers $\mathrm{C}_{2} \mathrm{~B}_{1} \mathrm{OH}_{12}$, dicarbo-closo-dodecaboranes. Here, we report the copper coordination polymer with the dicarboxylate of $1,7-$ dihydroxycarbonyl-1,7-dicarba-closo-dodecaborane [9]. The structure is similar with the reference [4].

The figure depicts a perspective of a part of structure unit of the complex. The environment of the $\mathrm{Cu}$ atom is five coordinated, in which two positions are occupied by the two oxygen atoms of the ligand carborane, one position is occupied by the oxygen atom of the water molecule, and the other two position are occupied by the two nitrogen atoms of the 1,10phenanthroline. The bond lengths of Cu1-01, Cu1-03 and Cu1-05 are $1.941 \AA$, $1.966 \AA$ and $2.245 \AA$, respectively. The bond lengths of Cu1-N1 and Cu1-N2 are 2.013 Å and 2.041 Å, respectively.

Acknowledgements: This work was supported by Henan science and technology project (Nos. 172102210161, 172102310166), Key Scientific Research Projects of Colleges and Universities, Henan Province (Nos. 18A150020) and Research Fund of Anyang Institute of Technology (Nos. YJJ2016014).

\section{References}

1. Bruker. SMART and SAINT for Windows NT Software Reference Manuals, Version 5.0. Bruker Analytical X-Ray Systems, Madison, WI (1997).

2. Sheldrick, G. M.: SADABS, a software for empirical absorption correction. University of Göttingen, Göttingen, Germany (1997).

3. SHELXL Reference Manual, version 5.1; Bruker Analytical X-Ray Systems, Madison, WI (1997).

4. Kahl, S. B.; Kasar, R. A.: Simple, high-yield synthesis of polyhedral carborane amino acids. J. Am. Chem. Soc. 118 (1996) 1223-1224.

5. Dou, D. M.; Zhang, D. P.; Li D. C.; Wang, D. Q.: Two type skeleton heterobimetallic trinuclear $\mathrm{Cu}-\mathrm{Mo}-\mathrm{S}$ clusters containing closo carborane diphosphine ligand 1,2-bis(diphenylphosphino)-1,2dicarba-closo-dodecaborane. Inorg. Chem. Commun. 9 (2006) 1109-1102.

6. Wang, H. W.; Li, D. C.; Hong, M.; Dou, J. M.: Syntheses, structural characterization and in vitro cytotoxic activity of triorganotin(IV) complexes based on 1,7-dihydroxycarbonyl-1,7-dicarbaclosododecaborane ligand. J. Organomet. Chem. 740 (2013) 1-9. 
7. Yang, L. G.; Wang, X; Gao, Q. Q.; Dai. Y. Q.: Crystal structure (7,8-bis(diisopropyl phosphino)-7,8-dicarba-nidoundecaborane- $\mathrm{K}^{2} P, P^{\prime}$ )-(benzoato- $\left.\mathrm{K}^{2} O, O^{\prime}\right)$ nickel(II), $\mathrm{C}_{21} \mathrm{H}_{42} \mathrm{~B}_{9} \mathrm{NiO}_{2} \mathrm{P}_{2}$. Z. Kristallogr. NCS. 232 (2017) 59-61.

8. Wang, X.; Yang, L. G.; Gao, Q. Q.; Dai. Y. Q.: Crystal structure of di- $\mu$-chlorido-bis[1,2-bis(dicyclohexylphosphino)-1,2- dicarba-closododecaborane- $\left.{ }^{2} P, P^{\prime}\right]$ zinc(II), $\mathrm{C}_{52} \mathrm{H}_{108} \mathrm{~B}_{20} \mathrm{C}_{12} \mathrm{P}_{4} \mathrm{Zn}_{2}$. Z. Kristallogr. NCS. 232 (2017) 75-77.

9. Herberhold, M.; Jin, G. X.; Yan, H.; Milius, W.; Wrackmeyer, B.: 16- and 18-Electron $C{ }^{\star}$ Rh complexes with 1,2-dicarba-closododecaborane(12)-1,2-dichalcogenolato ligands, as studied by multinuclear magnetic resonance. J. Organomet. Chem. 587 (1999) 252-257. 